\title{
Minimally invasive approach to lumbosacral decompression in a cadaveric canine model
}

\author{
B.T. Dent ${ }^{\mathrm{a}}$, G.T. Fosgate ${ }^{\mathrm{b}}$ \& B.F. Hettlich ${ }^{\mathrm{a}^{*}}$ \\ ${ }^{a}$ Department of Veterinary Clinical Sciences, College of Veterinary Medicine, The Ohio State University, 201 \\ Vernon Tharp Street, Columbus OH 43210, USA \\ ${ }^{b}$ Department of Production Animal Studies, Faculty of Veterinary Science, University of Pretoria, South Africa
}

\begin{abstract}
AIMS: To determine efficacy of a minimally invasive (MI) surgical approach using a human MI lumbar retractor for canine lumbosacral dorsal laminectomy and partial discectomy and to compare this technique to the standard open surgical (OS) approach.

METHODS: Lumbosacral dorsal laminectomy and partial discectomy was performed on 16 largebreed canine cadavers using either a standard OS $(n=8)$ or MI $(n=8)$ approach. Skin and fascial incision length, procedure time, and intraoperative complications were recorded. Postoperatively specimens were evaluated for laminectomy and discectomy dimensions, and visible damage to the cauda equina and exiting nerve roots.
\end{abstract}

RESULTS: Median length of skin and fascial incisions in the OS group were longer than in the MI group ( $p<0.001)$. Median laminectomy length was similar between both approaches $(p=0.234)$ but width was greater for the MI than OS approach $(p=0.002)$. Both approaches achieved similar partial discectomy width ( $p=0.279)$. Overall surgical time was longer for $\mathrm{MI}$ approaches compared to OS, with a median of 18.5 ( $\min 15.5, \max 21.8)$ minutes for MI compared to 14.6 ( $\min 13.1$, $\max 16.9$ ) minutes for OS $(p=0.001)$.

CONCLUSIONS: The MI approach reduced incision lengths while retaining comparable laminectomy and discectomy dimensions. For this in vitro model the $\mathrm{MI}$ approach required more time to complete, but this difference may not be relevant in clinical cases.

CLINICAL RELEVANCE: Dogs undergoing lumbosacral dorsal laminectomy are commonly large-breed dogs. The traditional open approach requires a large skin incision and soft tissue dissection, especially in overweight animals. A MI approach accomplishing the same surgical result while minimising soft tissue trauma could reduce post-operative pain and recovery time, and may lower wound-related complications. Clinical studies are needed to confirm postoperative benefit and assess operating times in vivo.

KEY WORDS: Dog, lumbosacral, dorsal laminectomy, minimally invasive surgery, lumbar retractor 
DLSS Degenerative lumbosacral stenosis

MI Minimally invasive

OS Open surgical

\section{Introduction}

Degenerative lumbosacral stenosis (DLSS) is a common condition of middle-aged and older large breed dogs, particularly German Shepherd and working dogs, leading to potentially debilitating pain and neurological deficits (Oliver et al. 1978; Danielsson and Sjöstrom 1999; Suwankong et al. 2008). Neurological dysfunction is related to compression of the cauda equina nerve roots secondary to pathophysiologic processes such as intervertebral disk protrusion, articular facet joint misalignment, and chronic lumbosacral instability (Lenehan and Tarvin 1998; De Risio et al. 2001; Benninger et al. 2006). In cases lacking proprioceptive deficits or incontinence, conservative therapy involving antiinflammatory drugs, weight reduction, and controlled exercise may be effective in controlling clinical signs (Janssens et al. 2009; Worth et al. 2009; Björn and Bergknut 2010).

More severe cases of DLSS usually require surgical intervention to decompress the cauda equina, most commonly via dorsal laminectomy with dorsal annulectomy and nucleus pulposus removal (Chambers 1989; Ness 1994; Janssens et al. 2000). Though various procedures have been described for treatment, traditionally such decompression is performed through an open dorsal approach to the lumbosacral junction, requiring a large skin incision and much soft tissue retraction to achieve adequate visualisation. (Chambers et al. 1988; Carozzo et al. 2008; O'Riordan et al. 2013) The amount of tissue trauma and potential patient morbidity can be substantial, particularly in obese dogs, as the approach dimensions must be adjusted to the depth of fatty tissue.

Minimally invasive (MI) surgery is becoming a standard of care for many human spinal surgeries, where iatrogenic trauma caused by the considerable soft tissue dissection and retraction has been well documented (Kawaguchi et al. 1994; Styf and Willen 1998; Gejo et al. 1999). Utilising unique retractors with visualisation aids, $\mathrm{MI}$ spinal surgery reduces incision dimensions and tissue dissection. MI spinal surgery in humans has been reported to decrease surgical complications, blood loss, iatrogenic soft-tissue injury, as well as post-operative pain and narcotic use, and a faster return to work (Mayer and Brock 1993; Foley and Lefkowitz 2002; Topcu et al. 2003). Though the discipline is more nascent in veterinary medicine, several studies have demonstrated the feasibility of $\mathrm{MI}$ spinal surgery and provided evidence of similar benefits (Wood et al. 2004; Leperlier et al. 2011; Lockwood et al. 2014).

The goal of this study was to develop a technique for and determine the efficacy of a MI approach to canine lumbosacral decompression using a human MI lumbar retractor. We hypothesised that the MI retractor could be used successfully in the large-breed canine lumbosacral spine to perform dorsal laminectomy and partial discectomy, and that the MI approach would significantly decrease soft tissue dissection while producing laminectomy and discectomy dimensions comparable to the open surgical (OS) approach. 


\section{Materials and methods}

The study was performed under an institutional animal care and use protocol. Sixteen entire fresh large-breed canine cadavers, subject to euthanasia for reasons unrelated to this study, were used. Dog breed, sex, body weight, body condition score, and subcutaneous fat depth were recorded. Lumbosacral vertebral columns were radiographed to assure physeal closure and lack of pre-existing disease. All surgical procedures were carried out by the same board-certified surgeon.

\section{Open surgical approach}

Eight dogs underwent standard open dorsal laminectomy and partial discectomy of the lumbosacral space (Sharp and Wheeler 2005; Lanz and Rossmeisl 2012; Dewey 2013). Dorsal midline skin incisions were made from the spinous process of the sixth lumbar vertebra to the mid-point of the sacral crest, as determined by palpation, and enlarged as needed to complete the procedures. Superficial and deep fascial layers were then incised and the surgical window was maintained with large angled Gelpi retractors. Epaxial muscle attachments on spinous processes of the seventh lumbar and first sacral vertebrae were elevated and the processes removed using rongeurs. The interarcuate ligament was sharply incised and removed and laminectomy was performed using a high-speed pneumatic drill with a $4 \mathrm{~mm}$ bur. The laminectomy was extended from the middle of the seventh lumbar to the second and third sacral vertebrae and laterally to the base of the articular processes. After exposure, the cauda equina was lateralised using a nerve hook and a partial discectomy was performed by removing the dorsal annulus fibrosus and nucleus pulposus using rongeurs and a triangular (No. 11) blade on a Bard-Parker No. 3 scalpel handle.

\section{Minimally invasive surgery approach}

Eight canine cadavers underwent MI dorsal laminectomy and partial discectomy of the lumbosacral space using a human MI lumbar retractor system (Pipeline Expandable Access System, Depuy Synthes, West Chester, PA, USA; Figure 1). The approach began with a small skin and fascial incision, approximately $3 \mathrm{~cm}$ in length, over the lumbosacral joint, as determined by palpation, through which a series of size-progressive dilators were inserted to expand the soft tissues. Once the surgical port was sufficiently enlarged, a lumbar retractor was fed over the dilators and into the incision, where it was locked in place by a rigid support arm. The telescoping retractor blades were extended through the epaxial musculature as needed to contact the vertebral laminae, after which a blade expander was utilised to distract the retractor blades. Once adequate visualisation of the surgical field was established (Figure 2), dorsal laminectomy and partial discectomy were carried out using the same protocol and instrumentation as in the OS group. 


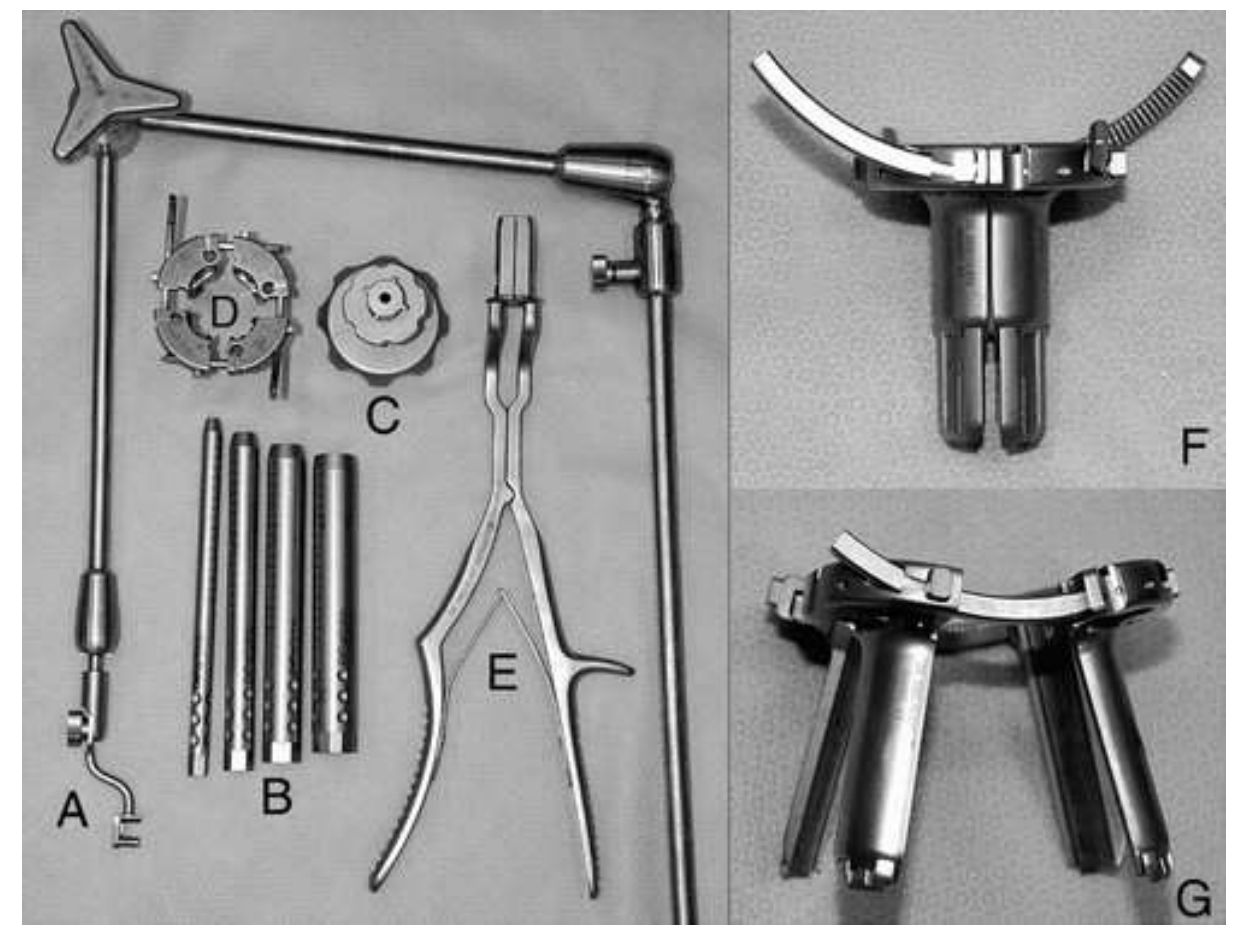

Figure 1. Minimally invasive lumbar retractor system (DePuy Synthes Pipeline Expandable Access System) including a flexible, self-locking table arm (A) to maintain retractor position intra-operatively; progressive dilators (B) to widen the access port made in the soft tissue; depth gauge (C) also used to lengthen the telescoping retractor blades; top view of the retractor (D); a retractor blades expander $(E)$, used to enlarge the deep access aperture; short retractor (F) with retractor arms closed and blades deployed; and long retractor (G) with retractor arms expanded and blades retracted. Note the arc of expansion increasing the distal working port while maintaining a relatively small skin incision.

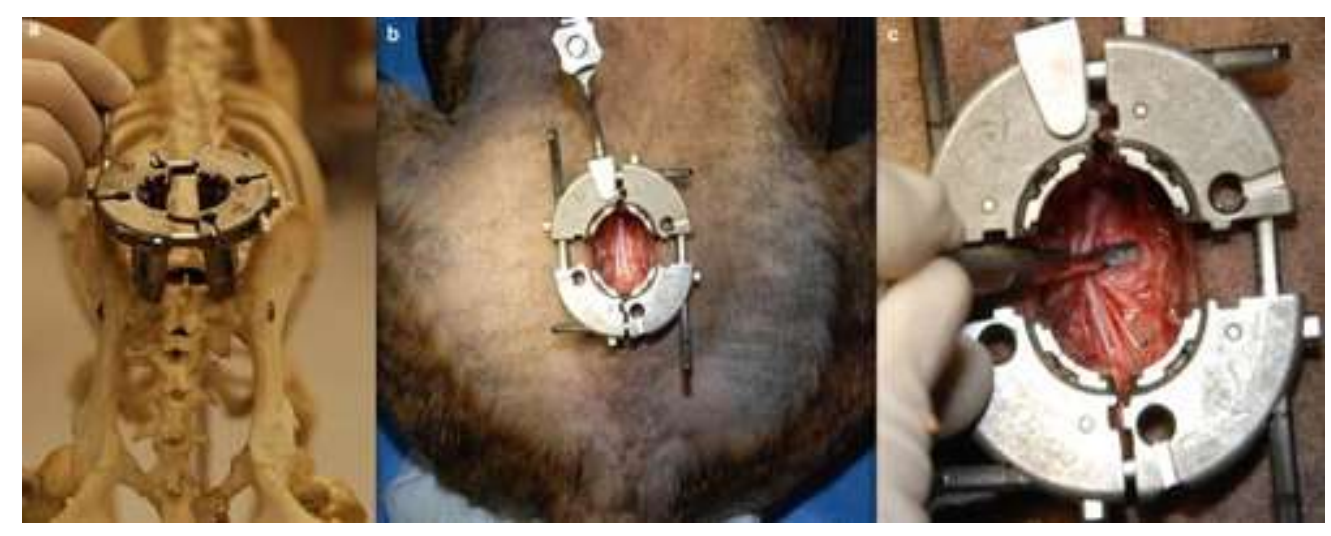

Figure 2. Photographs showing (a) position of a minimally invasive lumbar retractor used for dorsal laminectomy and partial discectomy over the canine lumbosacral space, (b) intra-operative view of the surgical site after dorsal laminectomy between the seventh lumbar and first sacral vertebrae had been performed, and (c) close-up view of the laminectomy site; the cauda equina has been lateralised to reveal the intervertebral discectomy site. 

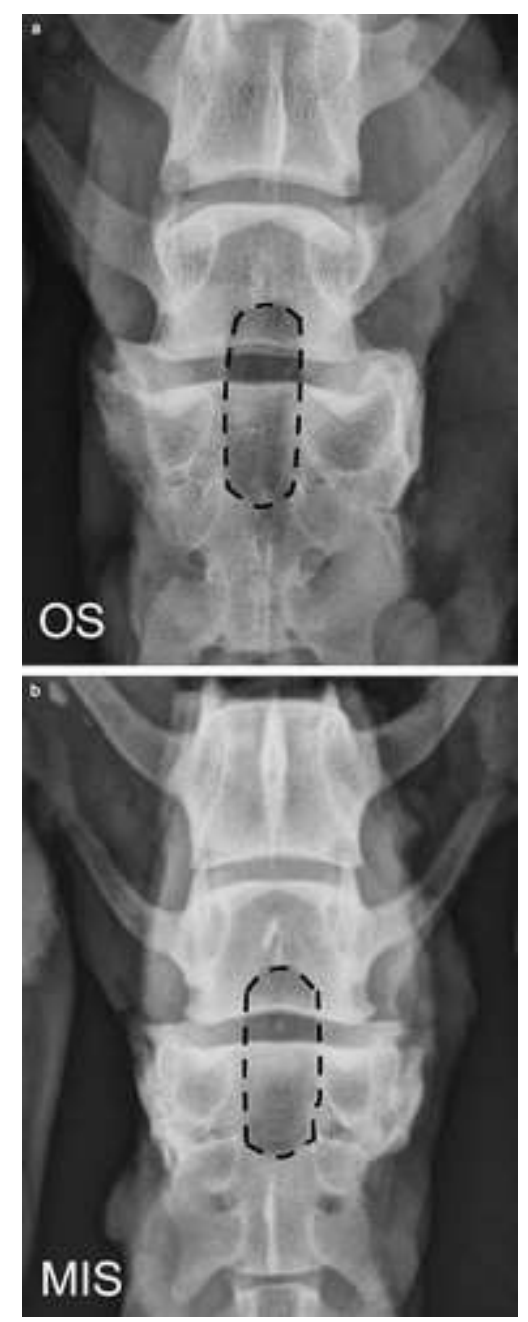

Figure 3. Dorsoventral radiographs of lumbosacral spines removed from canine cadavers following either (a) standard open or (b) minimally invasive surgical approaches for dorsal laminectomy and partial discectomy, demonstrating the laminectomy dimensions (dotted outline).

\section{Technique assessment}

Surgical time, procedural difficulties and adjustments, as well as intraoperative complications were recorded. Postoperative orthogonal lumbosacral radiographs were obtained to assess dimension of laminectomy and possible bony damage (Figure 3). Skin and fascial incision lengths were measured and the surgical site carefully inspected for iatrogenic neurovascular injury. Laminectomy length and width were recorded using digital calipers. The lower lumbar and sacral spine was then removed and sharply dissected at the seventh lumbar and first sacral vertebral disk space to allow assessment of the partial discectomy. Width of the partial discectomy defect was measured using calipers and compared to the total intervertebral disc width.

\section{Statistical analysis}

The normality assumption was evaluated for quantitative outcome data by plotting histograms, calculating descriptive statistics, and performing the Anderson-Darling test (MINITAB Statistical Software, Release 13.32, Minitab Inc, State College, PA, USA). Categorical data were compared 
between the $\mathrm{MI}$ and the OS groups using $\chi^{2}$ and Fisher exact tests. Quantitative data were compared between surgical groups using Mann-Whitney $U$ tests. Confounding by dog-level factors was investigated using a GLM but none was identified and only crude statistical comparisons are presented. Statistical evaluations of quantitative data were performed using IBM SPSS Statistics version 22 (International Business Machines Corp., Armonk, NY, USA).

\section{Results}

Dogs included in this study were pit bulls ( $n=10)$, pit bull cross $(n=3)$, one Labrador Retriever, one German Shepherd dog cross, and one mixed breed dog. Nine of the 16 dogs were male. There was no difference between groups in body weight, body condition score, depth of subcutaneous fat, gender, breed distribution, or intervertebral disk width $(p>0.1)$.

\section{Intraoperative assessment}

All OS procedures were completed to surgeon satisfaction with no apparent intraoperative complications. In the $\mathrm{MI}$ group, muscle creep underneath the retractor blades required adjustment of the $\mathrm{Ml}$ retractor in all cases. In five dogs, removal of the seventh lumbar spinous process prior to deploying the MI retractor arms facilitated better position of the retractor blades on the vertebral laminae of the seventh lumbar to first sacral vertebrae. The duration of all measured surgical procedures, with the exception of laminectomy, were longer in the MI than the OS approaches (Table 1).

Table 1. Median results for outcome measures following standard open (OS) or minimally invasive (MI) surgical approaches for dorsal laminectomy and partial discectomy assessed using canine cadavers.

\begin{tabular}{|c|c|c|c|c|c|}
\hline & & MI & & OS & \\
\hline Variable & $n$ & Median (min, max) & $n$ & Median (min, max) & P-value ${ }^{a}$ \\
\hline \multicolumn{6}{|l|}{ Duration (minutes) } \\
\hline Approach & 7 & $9.8(7.4,11.8)$ & 6 & $6.6(4.2,8.0)$ & 0.005 \\
\hline Laminectomy & 7 & $6.8(6.0,8.5)$ & 6 & $6.0(4.6,7.0)$ & 0.138 \\
\hline Approach + laminectomy & 7 & $16.9(14.2,20.2)$ & 8 & $12.3(10.1,14.4)$ & 0.001 \\
\hline Discectomy & |7 & $1.5(1.1,1.7)$ & 8 & $2.2(1.8,2.9)$ & $<0.001$ \\
\hline Total & ||7 & $18.5(15.5,21.8)$ & 8 & $14.6(13.1,16.9)$ & 0.001 \\
\hline \multicolumn{6}{|l|}{ Approach length (mm) } \\
\hline Skin & $\| 8$ & $55(49,67)$ & $\mid 8$ & $116(100,130)$ & $<0.001$ \\
\hline Fascia & 8 & $55(50,78)$ & 8 & $96(77,110)$ & $<0.001$ \\
\hline \multicolumn{6}{|l|}{ Laminectomy $(\mathrm{mm})$} \\
\hline Width & 8 & $11.6(10.0,13.0)$ & 8 & $9.8(8.8,10.7)$ & 0.002 \\
\hline
\end{tabular}




\begin{tabular}{|c|c|c|c|c|c|}
\hline & & MI & & OS & \\
\hline Variable & $n$ & Median (min, $\max$ ) & $n$ & Median (min, $\max$ ) & P-value ${ }^{a}$ \\
\hline Length & 8 & $31.7(28.2,35.1)$ & 8 & $29.1(24.4,33.5)$ & 0.234 \\
\hline \multicolumn{6}{|l|}{ Discectomy } \\
\hline Width (mm) & 8 & $6.6(4.4,8.7)$ & 8 & $7.1(5.2,9.0)$ & 0.279 \\
\hline Removed $(\%)^{b}$ & 8 & $27.0(19.2,39.1)$ & 8 & $30.6(21.6,40.9)$ & 0.195 \\
\hline
\end{tabular}

a Based on Mann-Whitney U tests.b Percentage of total intervertebral disc width.

\section{Post-operative assessment}

Anatomical dissection did not reveal any neurovascular damage from either approach. Length of skin and fascial incisions were reduced using the MI approach (Table 1). There was no difference in laminectomy length, however the $\mathrm{MI}$ approach produced wider laminectomies than the OS approach. Discectomy width as a percentage of total intervertebral disc width was similar in both approach groups (Table 1). In all dogs, minimal nuclear material remained within the intervertebral disc space.

\section{Discussion}

This study provides evidence of the efficacy of a MI approach to lumbosacral decompression in a canine cadaver model using a MI lumbar retractor system. Surgical site outcome measures were comparable or improved when dorsal laminectomy and partial discectomy were performed through the retractor portal and soft tissue approach dimensions were significantly reduced. The more limited approach reduced the degree of tissue manipulation and trauma, the most important means of controlling the surgical stress response, the constellation of local and systemic metabolic and hormonal changes following tissue injury (Kehlet 1997; Desborough 2000). This techniquedependent benefit should influence certain clinical outcomes, such as reduced haemorrhage, postoperative pain, wound complications, and recovery time, as has been demonstrated in numerous studies (Landreneau et al. 1993; Bosch et al. 2002; Foley and Lefkowitz 2002). In the case of DLSS, which commonly affects working dogs, the reduced post-operative morbidity may lead to faster return to service.

The MI approach extended the total surgical time compared to the OS approach (median $18.5 \mathrm{vs.}$ 14.6 minutes). This was mainly due to the need to reposition the Ml retractor during the soft tissue approach. However, it is difficult to make accurate suppositions regarding surgical time from cadaveric models. Surgeries performed in cadavers lack the patient-specific diseased surgical site, naturally occurring haemorrhage and other clinically relevant scenarios that can influence surgical time. In clinical cases, the additional few minutes added for MI retractor adjustments would probably not influence overall surgery time; however clinical studies will be required to confirm this.

Despite the improved outcomes seen through the use of a $\mathrm{Ml}$ approach, there were certain drawbacks to the adaptation of the lumbar retractor system to the canine lumbosacral spine. The 
first of the progressive tissue dilators has a trocar-style tip which, in pilot cases, traumatised the interarcuate ligament with possible damage to the cauda equina. The procedures included in this study were performed without the use of this instrument, opting instead to begin with a slightly larger, blunt dilator which was easily placed and did not lead to visible damage. Compared to the human lumbosacral spine, the relatively smaller canine lumbosacral anatomy presented a challenge, as the distance between spinous processes in many of the dogs did not allow placement of the retractor blades flush on the vertebral laminae. To counter this, in dogs where the spinous processes interfered, the seventh lumbar spinous process was removed with rongeurs after placement of the largest dilator prior to deploying the retractor. The majority of dogs in this study were lean, mediumsized pit bull mixes; however when the retractor was applied to the lumbosacral space of larger breeds, such as German Shepherd mixes, removal of seventh lumbar spinous process prior to deployment of the retractor blades was not necessary. Additionally, the blades of the retractor are flat at the distal end and allowed some muscle to slip back into the visual field. This tissue creep is also seen with human surgical applications. Tissue which could not be successfully retracted and was impairing visualisation of the dorsal vertebral lamina was excised in several MI procedures, but the amount of tissue excised was small. Tissue excision was not required in the OS approach. Although it is possible that the use of the retractor in cadaveric tissue may have compounded the issue, muscle creep would likely occur in clinical patients. Using retractor blades with a small, laterally deviated foot may decrease tissue creep.

Another limitation of the study was the cadaver population. Although similarity was maintained between groups in regards to size, age and breed, the cadaver population may not be representative of the clinical population. Dogs used in this study were typically young, lean, and healthy with apparently normal spines. Dogs with DLSS tend to be older with higher body condition score and spinal pathology. However, considering the cadaver experience with the retractor and typical DLSS patients, the utility of the lumbar retractor system for $\mathrm{Ml}$ approach may be more completely realised in larger, more obese dogs.

The ability to perform foramenotomy through the Ml approach was not assessed in this study but would be an important factor for the utility of this approach as many dogs affected by DLSS suffer from concurrent foramenal stenosis (Wood et al. 2004; Gödde and Steffen 2007).

In conclusion, the novel application of this retractor to the $\mathrm{MI}$ approach to the lumbosacral space in cadaveric dogs was successful. This retractor is widely available through Depuy Synthes throughout the global market and these initial results indicate its potential for adaptation to veterinary surgery. Clinical application of the retractor and post-operative patient follow-up will be required to confirm the proposed benefits of the $\mathrm{Ml}$ approach using such a retractor.

\section{Acknowledgements}

The authors would like to thank DePuy Synthes and specifically Darren Jackson for providing the Pipeline retractor for the duration of this study.

\section{References}

Benninger MI, Seiler GS, Robinson LE, Ferguson SJ, Bonél HM, Busato AR, Lang J. Effects of anatomic conformation on three-dimensional motion of the caudal lumbar and lumbosacral portions of the vertebral column of dogs. American Journal of Veterinary Research 67, 43-50, 2006 doi: 10.2460/ajvr.67.1.43 
Björn MP, Bergknut N. Degenerative Lumbosacral Stenosis in Dogs. Veterinary Clinics of North America: Small Animal Practice 40, 983-1009, 2010 doi: 10.1016/j.cvsm.2010.05.006

Bosch F, Wehrman U, Saeger HD, Kirch W. Laparoscopic or conventional cholecystectomy: Clinical and economic considerations. European Journal of Surgery 168, 5, 270-7, 2002 doi: 10.1002/ejs.45

Carozzo C, Cachon T, Genevois J, Fau D, Remy D, Daniaux L, Collard F, Viguier E. Transiliac approach for exposure of lumbosacral intervertebral disk and foramen: technique description. Veterinary Surgery 37, 27-31, 2008 doi: 10.1111/j.1532-950X.2007.00345.x

Chambers J. Degenerative lumbosacral stenosis in dogs. Veterinary Medical Report 1, 166-80, 1989

Chambers J, Selcer B, Oliver J. Results of treatment of degenerative lumbosacral stenosis in dogs by exploration and excision. Veterinary Comparative Orthopedics and Traumatology 3, 130-3, 1988

Danielsson F, Sjöstrom L. Surgical treatment of degenerative lumbosacral stenosis in dogs. Veterinary Surgery 28, 91-8, 1999 doi: 10.1053/jvet.1999.0091

De Risio L, Sharp NJ, Olby NJ, Muñana KR, Thomas WB. Predictors of outcome after dorsal decompressive laminectomy for degenerative lumbosacral stenosis in dogs: 69 cases (1987-1997). Journal of the American Veterinary Medical Association 219, 624-8, 2001 doi: 10.2460/javma.2001.219.624

Desborough JP. The stress response to trauma and surgery. British Journal of Anaesthesia 85, 1, 109-17, 2000 doi: $10.1093 / \mathrm{bja} / 85.1 .109$

*Dewey CW. Surgery of the Cauda Equina. In: Fossum TW (ed). Small Animal Surgery. Pp 1529-44. Mosby, St. Louis, MO, USA, 2013

Foley KT, Lefkowitz MA. Advances in minimally invasive spine surgery. Clinical Neurosurgery 49, 499-517, 2002

Gejo R, Matsui H, Kawaguchi Y, Ishihara H, Tsuji H. Serial changes in trunk muscle performance after posterior lumbar surgery. Spine 24, 1023-8, 1999 doi: 10.1097/00007632-199905150-00017 13. Gödde T, Steffen F. Surgical treatment of lumbosacral foraminal stenosis using a lateral approach in twenty dogs with degenerative lumbosacral stenosis. Veterinary Surgery 36, 705-13, 2007 doi: 10.1111/j.1532950X.2007.00324.x

Janssens LAA, Moens Y, Coppens P, Peremans K, Vinck H. Lumbosacral degenerative stenosis in the dog. Veterinary Comparative Orthopedics and Traumatology 13, 97-103, 2000

Janssens L, Beosier Y, Daems R. Lumbosacral degenerative stenosis in the dog. The results of epidural infiltration with methylprednisolone acetate: a retrospective study. Veterinary Comparative Orthopedics and Traumatology 22, 486-91, 2009

Kawaguchi Y, Matsui H, Tsuji H. Back muscle injury after posterior lumbar spine surgery. Part 2: Histologic and histochemical analyses in humans. Spine 19, 2598-602, 1994 doi: 10.1097/00007632-199411001-00018

Kehlet H. Multimodal approach to control post-operative pathophysiology and rehabilitation. British Journal of Anaesthesia 78, 606-17, 1997 doi: 10.1093/bja/78.5.606

Landreneau RJ, Hazelrigg SR, Mack MJ, Dowling RD, Burke D, Gavlick J, Perrino MK, Ritter PS, Bowers CM, DeFino J, Postoperative pain-related morbidity: Video-assisted thoracic surgery versus thoracotomy. The Annals of Thoracic Surgery 56, 1285-9, 1993 doi: 10.1016/0003-4975(93)90667-7 
*Lanz OI, Rossmeisl JH Jr. Lumbosacral Spine. In: Tobias KM, Johnston SA (eds). Veterinary Surgery: Small Animal. Pp 476-86. Saunders, St. Louis, MO, USA, 2012 20. *Lenehan T, Tarvin G. Surgical treatment of cauda equina compression syndrome by laminectomy. In: Bojrab M (ed). Current Techniques in Small Animal Surgery. Pp 859-61. Lea \& Febiger, Philadelphia, PA, USA, 1998

Leperlier D, Manassero M, Blot S, Thibaud JL, Viateau V. Minimally invasive video-assisted cervical ventral slot in dogs: a cadaveric study and report of 10 clinical cases. Veterinary Comparative Orthopedics and Traumatology 24, 50-6, 2011 doi: 10.3415/VCOT-10-04-0066

Lockwood AA, Griffon DJ, Gordon-Evans W, Matheson JA, Barthélémy N, Schaeffer DJ. Comparison of two minimally invasive approaches to the thoracolumbar spinal canal in dogs. Veterinary Surgery 43, 209-21, 2014 doi: 10.1111/j.1532-950X.2014.12098.x

Mayer HM, Brock M. Percutaneous endoscopic discectomy: surgical technique and preliminary results compared to microsurgical discectomy. Journal of Neurosurgery 78, 216-25, 1993 doi: 10.3171/jns.1993.78.2.0216

Ness M. Degenerative lumbosacral stenosis in the dog: A review of 30 cases. Journal of Small Animal Practice 35, 185-90, 1994 doi: 10.1111/j.1748-5827.1994.tb01683.x

Oliver JE Jr, Selcer RR, Simpson S. Cauda equina compression from lumbosacral malarticulation and malformation in the dog. Journal of the American Veterinary Medical Association 173, 207-14, 1978

O'Riordan J, Moisoonnier PHM, Kirby BM. Ventral surgical approach to the lumbosacral joint in the dog. Veterinary Surgery 42, 85-90, 2013 doi: 10.1111/j.1532-950X.2012.01070.x

*Sharp NJ, Wheeler SJ. Small Animal Spinal Disorders: Diagnosis and Surgery. Pp 181-202. Mosby, St. Louis, MO, USA, 2005

Styf JR, Willen J. The effects of external compression by three different retractors on pressure in the erector spine muscles during and after posterior lumbar spine surgery in humans. Spine 23, 354-8, 1998 doi: 10.1097/00007632-199802010-00014

Suwankong N, Meij BP, Voorhout G, de Boer AH, Hazewinkel HA. Review and retrospective analysis of degenerative lumbosacral stenosis in 156 dogs treated by dorsal laminectomy. Veterinary Comparative Orthopedics and Traumatology 21, 285-93, 2008

Topcu O, Karakayali F, Kuzu MA, Ozdemir S., Erverdi N., Elhan A., Aras N. Comparison of long-term quality of life after laparoscopic and open cholecystectomy. Surgical Endoscopy 17, 2, 291-5, 2003 doi: 10.1007/s00464001-9231-2

Wood BC, Lanz OI, Jones JC, Ozdemir S, Erverdi N, Elhan A, Aras N. Endoscopic-assisted lumbosacral foraminotomy in the dog. Veterinary Surgery 33, 221-31, 2004 doi: 10.1111/j.1532-950X.2004.04033.x

Worth AJ, Thompson DJ, Hartman AC. Degenerative lumbosacral stenosis in working dogs: Current concepts and review. New Zealand Veterinary Journal 57, 319-30, 2009 doi: 10.1080/00480169.2009.64719 